\title{
EXPERIENCIAS DE VINCULACIÓN SOCIAL UNIVERSITARIA: motivaciones para la participación estudiantil
}

\author{
Adriana Yanina Ortiz ${ }^{1}$
}

\section{RESUMEN}

Por qué las personas se implican en voluntariado y qué sustenta la acción voluntaria son inquietudes fundamentales en la investigación motivacional. Este artículo examina las motivaciones de estudiantes de las Facultades de Ciencias de la Salud y Medicina, de las Universidades Nacionales de Salta y Tucumán, en Argentina, que participan en proyectos de Vinculación Social Universitaria. Conocer cuáles son los procesos motivacionales que subyacen a la conducta de participación voluntaria puede contribuir a comprender las expectativas individuales en torno a las prácticas extensionistas en la universidad, y a repensar las estrategias que comúnmente se utilizan para convocar y para lograr la permanencia de los estudiantes en los diferentes proyectos. Los datos fueron obtenidos a partir de un análisis documental de informes técnicos finales, de entrevistas a voluntarios y del relato de experiencias de los participantes. Se optó por un análisis de contenido para operar sobre el contenido temático de los documentos y lograr una representación de la información tratada. El proceso de categorización reflejó seis motivaciones generales y nueve subcategorías específicas. Los resultados destacan una media de dos motivos para ser voluntario y la prevalencia de "Expresión de Valores" junto con "Conocimiento", "Desarrollo Profesional", y "Realización Personal" sobre las categorías restantes. Se considera que detrás de la acción voluntaria coexisten hasta dos motivos distintos que llevan a los estudiantes a implicarse y permanecer en los proyectos por largos períodos de tiempo, y que esta acción es movilizada, sobre todo, por una preocupación genuina por los otros.

Palabras clave: Vinculación social universitaria. Motivación. Estudiantes universitarios.

\footnotetext{
1 Doctora en Educação, especialidad em Psicología de la Educación (Universidade de Lisboa, PT). Becaria del PNPD/CAPES, Programa de Pós-Graduação em Gestão do Conhecimento nas Organizações (PPGGCO), del Centro Universitário de Maringá UniCesumar, PR-Brasil. Investigadora en INIPE, Facultad de Humanidades, Universidad Nacional se Salta, Argentina. Investigadora en UIDEF, Instituto de Educação, Universidade de Lisboa, Portugal. E-mail: ortizadrianayanina@gmail.com ORCID: http://orcid.org/0000-00020460-1036
} 


\title{
EXPERIÊNCIAS DE VINCULAÇÃO SOCIAL UNIVERSITÁRIA: motivações para a participação dos estudantes
}

\section{RESUMO}

Por que as pessoas se envolvem em voluntariado e o que sustem a ação voluntária são inquietudes fundamentais na investigação motivacional. Este artigo examina as motivações de estudantes das Faculdades de Ciências da Saúde e Medicina, das Universidades de Salta e Tucumán, em Argentina, que participam em projetos de Vinculação Social Universitária. Conhecer quais os processos motivacionais que subjazem à conduta de participação voluntária pode contribuir a compreender as expetativas individuais em torno às práticas extensionistas na universidade, e a repensar as estratégias que comumente se utilizam para convocar e para lograr a permanência dos estudantes nos diferentes projetos. Os dados foram obtidos a partir de uma análise documental de informes técnicos finais, de entrevistas a voluntários e do relato de experiência dos alunos. Optou-se por uma análise de conteúdo para operar sobre o conteúdo temático dos documentos e lograr uma representação da informação tratada. O processo de categorização refletiu seis motivações gerais e nove subcategorias específicas. Os resultados destacam uma média de dois motivos para ser voluntário e a prevalência de "Expressão de Valores, junto com "Conhecimento", "Desenvolvimento Profissional" e "Realização Pessoal" sobre as categorias restantes. Considera-se que por trás da ação voluntária coexistem até dois motivos distintos que levam aos estudantes se envolverem e permanecer nos projetos por longos períodos do tempo, e que esta ação é mobilizada, sobre tudo, por uma preocupação genuína pelos outros.

Palavras-chave: Vinculação social universitária. Motivação. Estudantes universitários.

\section{UNIVERSITY SOCIAL OUTREACH EXPERIENCES:}

\section{motivations for student engagement}

\begin{abstract}
Why people are involved in volunteering and what sustains voluntary action are fundamental concerns in motivational research. This article examines the motivations of students of the Faculties of Health Sciences and Medicine of the National Universities of Salta and Tucumán, Argentina, who participate in university social outreach projects. Knowing which the motivational processes that underlie the behavior of voluntary participation are can help understand the individual expectations about extension practices in the university and rethink the strategies that are commonly used to convene and to achieve the permanence of students in the different projects. The data was obtained from a documentary analysis of final technical reports, interviews with volunteers and the experiences of the participants. We carried out a content analysis to operate on the thematic content of the documents and achieve a representation of the information treated. The categorization process reflected six general motivations and nine specific subcategories. The results highlight two reasons for volunteering and the prevalence of "Expression of Valves" along with "Knowledge", "Professional Development", and "Personal Realization" as remaining categories. It is considered that behind voluntary engagement action there coexist up to two different reasons that lead students to
\end{abstract}


get involved in projects for long periods of time and that this action is mobilized, above all, by a genuine concern for others.

Keywords: Linking social university. Motivation. University student.

\section{INTRODUCCIÓN}

El presente artículo se deriva de una investigación de mayor alcance 2 que busca analizar la experiencia de trabajo de equipos universitarios en sus esfuerzos por articular actividades de vinculación social (extensión y voluntariado), docencia e investigación, así como también indagar de qué manera estas experiencias contribuyen a la mejora de la calidad de vida de las comunidades en que se desarrollan, a la formación de los estudiantes universitarios y a las labores de docencia e investigación.

Específicamente este artículo focaliza en los estudiantes universitarios que participan en proyectos de extensión y voluntariado, y en los procesos psicológicos que subyacen a la decisión de ser voluntarios, con el fin de comprender las expectativas individuales que giran en torno a las prácticas extensionistas en la universidad, y repensar las estrategias que comúnmente se utilizan para convocar y lograr la permanencia de los estudiantes en los diferentes proyectos por largos períodos de tiempo.

El estudio de las motivaciones para el voluntariado tiene una larga tradición en la investigación (ALLISON et al., 2002; CHACÓN et al., 2010; CLARY et al., 1998; CNAAN, GOLDBERG-GLEN, 1991; FIGUEIRA, 2013; GIDRON, 1978; HOLDSWORTH, 2010; ORTIZ, 2013) y se presenta como objeto de estudio de gran atractivo para los teóricos e investigadores que intentan comprender cuáles son los procesos psicológicos que se activan y se desarrollan en la conducta voluntaria y desinteresada de una persona por ayudar a otros, sin esperar ni recibir alguna recompensa a cambio.

\footnotetext{
2 Este artículo presenta algunos resultados del proyecto de investigación "Vinculación social universitaria, calidad de vida de la población, formación de los estudiantes, e investigación. Logros, dificultades y desafíos en las experiencias de equipos universitarios del NOA en el área de salud", que la autora realizó en tanto becaria postdoctoral del Consejo Nacional de Investigaciones Científicas y Técnicas (CONICET) en la Universidad Nacional de Salta (UNSa), Argentina, contando para ello con el apoyo institucional de la UNSa y la Universidad Nacional de Tucumán, y con el financiamiento del CONICET.
} 
Las preguntas en relación a por qué las personas son voluntaria y qué sustenta la acción voluntaria son inquietudes fundamentales en la investigación motivacional (CLARY et al., 1998). El voluntariado aparece como un tipo de ayuda específico, que exige una planificación considerablemente mayor a la de una colaboración espontánea, y que implica la clasificación de prioridades y la adecuación de las capacidades e intereses personales con un determinado tipo de intervención (CLARY; SNYDER, 1999). De acuerdo con ello, la persona voluntaria busca oportunidades para ayudar a los demás, reflexiona y decide -racional y deliberadamente- sobre su participación en proyectos de voluntariado, a partir del grado en que determinadas actividades puedan ofrecer una respuesta a sus necesidades personales (HOULE et al., 2005).

El Enfoque Funcional del Voluntariado (CLARY et al., 1998) propone una perspectiva que es explícitamente concerniente con las razones y los propósitos que subyacen a los fenómenos psicológicos. Uno de sus postulados centrales sostiene que una acción puede estar al servicio de diferentes funciones psicológicas, y ofrece explicaciones para entender por qué muchas personas participan en una misma actividad, cuando los motivos por los cuales se implican son significativamente diferentes. Este enfoque sugiere, además, que importantes eventos psicológicos dependerán del grado de coincidencia entre las inquietudes motivacionales de los indviduos con situaciones que pudiesen satisfacerlas (CLARY; SNYDER, 1999).

Las proposiciones centrales de esta perspectiva tienen que ver, por un lado, con el hecho de que la decisión de involucrarse en actividades de voluntariado -que aparenta ser similar entre los sujetos- puede reflejar marcadas diferencias en los procesos motivacionales subyacentes $y$, por otro lado, con la idea fundamental de que las funciones psicológicas a las que da respuestas el voluntariado se manifiestan en la dinámica del desarrollo de esta forma particular de ayudar, e influyen fuertemente en las decisiones relacionadas con la iniciación y la continuidad de dicha conducta. 
Este enfoque ha permitido el desarrollo de cuestionarios cerrados para estudiar las motivaciones para el voluntariado. El más utilizado en la última década es el Volunteer Functions Inventory (VFI), elaborado por Clary y colaboradores (1998), que teoriza seis motivaciones explicativas acerca de por qué una persona es voluntaria (tabla 1). La primera que destaca es la motivación Valores (values), centrada en el interés humanitario y altruista por ayudar a otros. En segundo lugar, Comprensión o Conocimiento (understanding), refleja el gusto por aprender sobre temas diversos y descubrir el mundo a través del voluntariado, esto es, las personas pueden desarrollar competencias, habilidades y adquirir nuevos conocimientos en contacto con la realidad mediante el voluntariado. Una tercera motivación refiere al Ajuste Socia (social) que responde a la necesidad de adaptarse a una norma social subjetiva, es decir, a lo que las personas cercanas del sujeto valoran como importante; en este sentido, si para la familia o los amigos el voluntariado representa una actividad valiosa, la participación de la persona podría estar motivada por esa mirada positiva y asertiva que su entorno le adjudica a la práctica voluntaria. Carrera (carreer) expresa la búsqueda de beneficios para el desarrollo de una carrera profesional por lo que un voluntario puede ver que el ambiente donde desarrolla las actividades podría favorecer su futuro profesional. Autoestima (enhancement) refiere a la motivación centrada en el interés por el disfrute y la generación de sentimientos positivos que produce la participación en actividades voluntarias. Por último, Protección (protective) atiende a una necesidad de defensa del "yo" de situaciones desagradables, o de evitación de sentimientos negativos tales como la soledad o la depresión; la práctica de voluntariado, por lo tanto, podría estar motivada por esta necesidad psicológica de autoprotección.

El VFI ha sido ampliamente utilizado en la investigación y en los más variados contextos (ALLISON et al., 2002; BRUYERE, RAPPE, 2007; CLARY, SNYDER, 1991; CLARY et al., 1996; CLARY et al., 1998; DÁVILA, DÍAZ-MORALES, 2009; FIGUEIRA, 2013; FINKELSTEIN, 2009; HOULE et al., 2005; OKUN, SCHULTZ, 2003; ORTIZ, 2013; WU et al., 2009). Chacón et al. (2017) desarrollaron una 
revisión sistemática de las investigaciones sobre voluntariado que hayan usado el VFI de Clary et al. (1998), y encontraron que de un total de 48 estudios revisados, el factor Valores ha obtenido la mayor puntuación media en general, mientras que las puntuaciones más bajas corresponden a las motivaciones Carrera y Protección o Defensa del Yo. En el caso de estudios realizados con muestras cuya edad media es menor a 40 años, las motivaciones Carrera y Conocimiento obtienen las puntuaciones más altas. La revisión de estos autores destaca también un dato importante en relación con la estructura factorial del VFI, puesto que de 26 investigaciones que han llevado a cabo un análisis factorial, 18 de ellas confirman la estructura original de seis factores.

TABLA 1 - Categorías Motivacionales según el VFI

\begin{tabular}{c}
\hline Valores \\
\hline Comprensión \\
\hline Ajuste Social \\
\hline Carrera \\
\hline Realización Personal \\
\hline Protección \\
\hline
\end{tabular}

Fuente: Clary et al. (1998).

El principal inconveniente de este tipo de instrumentos, según Chacón y colaboradores (2010), es que las personas señalan como motivaciones lo que en realidad podrían ser expectativas, es decir, puntúan en todos aquellos ítems que componen el cuestionario, aunque en principio no todos ellos determinen la decisión de convertirse en voluntarios. Algunos autores han cuestionado el uso indiscriminado de este instrumento para evaluar las motivaciones (ALLISON et al., 2002; CHACÓN et al., 2010), y han abogado por la utilización de una pregunta abierta por medio de la cual los individuos pueden aportar una explicación que justifique por qué son voluntarios. En este sentido, el estudio de Allison et al. (2002) confirma que, además de las seis motivaciones teorizadas por Clary y colaboradores (1998), los voluntarios mencionan tres motivos más: disfrute, religiosidad y trabajo en equipo. 


\section{ISSN 2237-9460}

Por su parte, Chacón et al. (2010) han encontrado que, en general, los voluntarios manifiestan una media de dos motivos y emergen motivaciones adicionales que no están contempladas en los cuestionarios (ver tabla 2). Sus resultados de investigación mencionan, en primer lugar, la motivación Valores con las subcategorías Valores Religiosos, de Transformación Social, de Reciprocidad y de Interés por la Comunidad; dentro de esta última se distinguen valores de ayuda a un territorio específico y valores de ayuda a un colectivo específico. En segundo lugar, aparece la motivación Conocimiento-Comprensión con una sub-categoría: autoconocimiento. En tercer, cuarto y quinto lugar se mencionan las motivaciones para el Ajuste Social, Mejora del Currículum y Defensa del yo. Una sexta motivación refiere al Mejoramiento de la Estima, que presenta cuatro subcategorías: estima, crecimiento personal, relaciones sociales y disfrute. La séptima categoría motivacional alude al Compromiso Organizacional que incluye compromiso institucional y con el grupo. Interés en la Actividad, con las subcategorías interés en la actividad específica e interés en la actividad con personas, conforman la octava categoría. La motivación Condiciones incluye respuestas que no fueron propiamente motivos sino más bien condiciones o requisitos necesarios que favorecen o facilitan la realización del voluntariado. Y, por último, la décima categoría que los autores han elaborado agrupa respuestas poco específicas, que no pudieron ser clasificadas dentro de las categorías previas.

TABLA 2 - Categorías Motivacionales según Chacón eł al. (2010)

\begin{tabular}{|c|c|c|}
\hline \multirow{5}{*}{ Expresión de valores } & \multicolumn{2}{|l|}{ Valores religiosos } \\
\hline & \multicolumn{2}{|c|}{ Valores de Transformación Social } \\
\hline & \multicolumn{2}{|l|}{ Valores de Reciprocidad } \\
\hline & \multirow{2}{*}{$\begin{array}{l}\text { Valores de interés por la } \\
\text { Comunidad }\end{array}$} & $\begin{array}{l}\text { Valores de Ayuda a un } \\
\text { Territorio Específico }\end{array}$ \\
\hline & & $\begin{array}{l}\text { Valores de Ayuda a un } \\
\text { Colectivo Específico }\end{array}$ \\
\hline Conocimiento & \multicolumn{2}{|l|}{ Autoconocimiento } \\
\hline
\end{tabular}


Mejora del Currículum

Defensa del yo

\begin{tabular}{ll}
\hline \multirow{2}{*}{ Mejora de la Estima } & Estima \\
\cline { 2 - 2 } & Crecimiento Personal \\
\cline { 2 - 2 } & Realciones Sociales \\
\hline Compromiso & Disfrute \\
\hline Organizacional & Compromiso Institucional \\
\hline Interés en la Actividad & Interés en la Actividad específica \\
\hline & Interés en la Actividad con \\
\hline
\end{tabular}

Condiciones

Otros

Fuente: Elaboración de la autora a partir de Chacón et al. (2010)

Atendiendo a las motivaciones propuestas por Chacón et al. (2010), consideradas como categorías a priori, el objetivo de este artículo es analizar las motivaciones de estudiantes universitarios que participan en proyectos de vinculación social universitaria en la Universidad Nacional de Salta (UNSa) y en la Universidad Nacional de Tucumán (UNT). Tanto la UNSa como la UNT son universidades públicas y están situadas en las ciudades capitales de las provincias de Salta y Tucumán, en la región Noroeste de Argentina. Con la finalidad de orientar al lector, es preciso describir y caracterizar, brevemente, los cuatro proyectos, que son el marco contextual de esta investigación.

"Promoviendo el derecho a la salud sexual integral en comunidades originarias- Nawo lahjata ta iyek salud sexual integral ta inamejhen" -en adelante referido con las siglas SSI- es un proyecto de voluntariado desarrollado por el Servicio de Orientación y Tutorías de la Facultad de Ciencias de la Salud (UNSa), dirigido por la Lic. Alejandra Bergagna. Tiene por objetivo promover la salud sexual en jóvenes de la comunidad Wichi de La Puntana, Santa Victoria Este, Departamento Rivadavia, Provincia de Salta. Específicamente busca conocer las creencias, saberes y prácticas sobre salud sexual y reproductiva de las comunidades originarias, identificar desde 
su cosmovisión los aspectos que promueven la salud sexual y reproductiva y aquellos que son considerados como problemas, y generar acciones/espacios de intervención para la promoción de la salud sexual integral desde su propia cultura. La población destinataria son jóvenes y adolescentes de 16 a 24 años, que asisten a las escuelas primaria y secundaria, y algunos jóvenes no escolarizados. Conforman el equipo de trabajo diez personas entre estudiantes y docentes. Tres docentes del área de sociales, tres enfermeros y cuatro estudiantes universitarios, tres de los cuales son miembro integrante del Centro de Estudiantes Universitarios de Pueblos Originarios (CEUPO). La implicación de los estudiantes es plenamente voluntaria.

Una segunda experiencia es tomada del proyecto "Apoyo a la Lactancia Materna" -en adelante ALM- de la Cátedra de Bioquímica de la Facultad de Medicina (UNT), cuya directora responsable es la Dra. Silvina Aguirre. A partir de los bajos índices de lactancia que existe a nivel mundial, nacional, provincial y regional, este proyecto se propone la promoción de la lactancia materna en la comunidad. Las destinatarias son madres que asisten a la Maternidad, al Hospital San Pablo, Hospital de Niños y al Centro de Atención Primaria de la Salud (CAPS, Yerba Buena), en la Provincia de Tucumán. En estos sitios, cuatro docentes y un total aproximado de treinta alumnos, cuya participación es voluntaria, desarrollan año a año talleres y consejerías personalizadas con la finalidad de crear conciencia acerca de la importancia que tiene la leche materna durante los primeros meses de vida de los bebés.

El proyecto "Control de Salud del Varón Adulto" -en adelante referido con las siglas CSA- está a cargo de la Dra. Analía Soria de González quien es también responsable por la Cátedra de Patología Molecular de la Facultad de Bioquímica, Química y Farmacia y de la Unidad de Práctica Final Obligatoria de la Facultad de Medicina (UNT). El objetivo de este proyecto es promover el control periódico de salud en la población de adultos mayores de 50 años de edad, en las comunidades de Graneros, San Pablo, Garmendia, Los Pereiras, en la Provincia de Tucumán. Entre las actividades se 
incluyen promoción, educación para la salud, detección temprana de enfermedades y gestión de casos. Participan de este proyecto un grupo aproximado de quince docentes y cuarenta estudiantes que cursan las carreras de bioquímica, odontología y medicina. En el caso de los dos primeros, la participación es voluntaria, mientras que para los estudiantes de medicina la experiencia es obligatoria, pues forma parte de la práctica final de la carrera de médico.

Finalmente, la Dra. Liliana Fracchia a través de la Cátedra de Metodología de la Investigación de la Facultad de Medicina (UNT), es responsable del proyecto "Nutrición, salud mental y embarazo" que se desarrolla junto con el Instituto de Maternidad y Ginecología Ntra. Sra. de las Mercedes-Sistema Provincial de Salud (SiProSa) Ministerio de Salud de la Provincia de Tucumán. Participan diez docentes y diecisiete estudiantes voluntarios de las carreras de psicología, trabajo social, medicina, nutrición y bioquímica. Dado que el embarazo confiere a la mujer una mayor vulnerabilidad para la aparición de trastornos físicos y psicológicos, el proyecto busca implementar estrategias de asesoramiento nutricional y contención emocional a madres que concurren a la maternidad, desde el embarazo hasta los primeros meses posparto. Los cambios bioquímicos y sociales ligados a la emocionalidad de la gestante pueden llevarla a no dar la importancia debida a su nutrición $y$, consecuentemente, generar problemas en la relación psicofisiológica con el bebé, por lo que la prevención, detección y tratamiento de estos problemas es uno de los objetivos prioritarios de este equipo de trabajo.

La práctica de voluntariado encarada desde la Universidad, además de ser un aporte significativo para la sociedad, se transforma en espacio de práctica pre-profesional, de aprendizaje significativo, donde teoría y empiria dialogan, y los contenidos académicos pueden aprenderse en interacción con contenidos derivados de una realidad social próxima (ARIAS, TARZIBACHI, 2007; NICHOLLS, SCHIMMEL, 2012; TAPIA, 2008). Estas actividades parecen adquirir cada vez más relevancia en las universidades y los 
estudiantes son invitados a participar en ellas y a comprometerse como actores educativos y agentes comunitarios (FIGUEIRA, 2013; ORTIZ, 2013).

De acuerdo con la información aportada por las docentes responsables de los proyectos, un total aproximado de 91 estudiantes universitarios se implican en estas cuatro propuestas de vinculación social, alumnos que no sólo tienen antecedentes de participación, es decir, no es el primer año que forman parte del equipo, sino que además se proyectan continuar acompañando al grupo por tiempo indeterminado.

La particularidad de estos alumnos (a excepción de los estudiantes de medicina del proyecto CSA), está en la decisión personal de participar. Son voluntarios porque quieren, no son obligados ni lo hacen como requisito para aprobar alguna asignatura. Esta característica identitaria de los voluntarios (ORTIZ, 2013) conduce a un cuestionamiento acerca de los procesos motivacionales que subyacen a la acción voluntaria. En este sentido interesa indagar cuáles son los procesos psicológicos y sociales que están por detrás de la acción de las personas, y que pueden explicar la decisión de iniciar y sustentar la participación en proyectos de voluntariado, extensión o vinculación social universitaria.

\section{METODOLOGÍA}

En el marco de la tradición cualitativa de investigación, dadas las características de la población y de los proyectos objeto de estudio, se ha desarrollado una investigación documental que implicó un proceso sistemático y secuencial de recolección, selección, clasificación, evaluación y análisis de contenido de material empírico impreso y gráfico, que sirvió como fuente conceptual, teórica y metodológica para el presente estudio.

\section{Participantes}

La muestra inicial estaba compuesta por 34 estudiantes que participan como voluntarios en los proyectos referidos. No obstante dado que el relato de experiencia de dos de ellos no ofrecía información sustantiva en relación con el objetivo propuesto para esta investigación, la muestra final quedó 
conformada por un total de 32 estudiantes de las carreras de medicina, psicología, trabajo social, nutrición, odontología y bioquímica de la UNT, y de enfermería y ciencias de la educación de la UNSa. La muestra es mayoritariamente femenina (78\%) y, de acuerdo con lo informado por las directoras de los proyectos, las edades varían entre 18 y 25 años.

\section{Instrumento}

Los datos fueron obtenidos a partir de un análisis documental de informes técnicos, de entrevistas a voluntarios y del relato de experiencias de los participantes de los proyectos de voluntariado y extensión. Estos últimos fueron recuperados de los Informes Técnicos de Avance y Final que cada equipo debe presentar a la Secretaría de Políticas Universitarias (SPU), una vez concluido el plazo de ejecución del proyecto.

Se optó por un análisis de contenido para operar sobre el contenido temático de los documentos y lograr una representación de la información tratada. Para la indización se utilizaron descriptores, es decir, términos normalizados, formalizados y homologados en un lenguaje documental, empleados para representar, sin ambigüedad, los conceptos tratados en los documentos.

\section{Procedimiento}

El proceso de categorización consistió en la elaboración de un listado teórico que incluía las motivaciones identificadas por Chacón et al. (2010), donde están también contempladas las categorías propuestas por Clary y colaboradores (1998) y Allison et al. (2002). Sin considerar "condiciones" y "otros", en total fueron 23 categorías motivacionales definidas a priori.

De los datos aportados por los estudiantes fue posible identificar un total de 86 motivos. Las respuestas fueron clasificadas de acuerdo al grado de adecuación a cada categoría preestablecida y considerando, además, las subcategorías propuestas por los autores, debido a la frecuencia de aparición de algunas respuestas que no se correspondían exactamente con una definición general. 


\section{RESULTADOS}

El proceso de categorización resultó en la estructura que se observa en la Tabla 3. De cada categoría se propone una definición operacional y se presentan, además, algunos fragmentos de las respuestas de los estudiantes que dieron lugar a la conformación de cada una de ellas.

\section{Expresión de Valores}

Esta categoría incluye todas las expresiones que explicitan valores sociales de interés altruista, humanitario o heterocentrado. Entre las más frecuentes se destacan "para dar servicio a los demás", "por solidaridad", "por hacer algo útil", "para retribuir a la sociedad su apoyo". Expresión de valores presenta, además, dos subcategorías: valores de reciprocidad y valores de interés por la comunidad.

1.1. Valores de Reciprocidad. Esta subcategoría responde a la norma social de reciprocidad de forma genérica o específica. Algunas respuestas expresan claramente que los estudiantes participan en los voluntariados con el fin de retribuir, devolver o agradecer algo que han recibido, mientras que otras están más centradas en el interés por el intercambio, en el dar para recibir algún beneficio a cambio del tiempo y los atributos personales puestos a disposición en las prácticas del voluntariado. Expresan los voluntarios: "una actividad en la que además de devolver a la sociedad parte de los conocimientos adquiridos en la facultad nos permita seguir aprendiendo con los demás, nos interesó sobre manera" (Rocío-NSE); "(...) devolver un poco de ese apoyo que nos brinda la sociedad con la posibilidad de estudiar en una Universidad Pública" (Sergio-NSE).

TABLA 3 - Motivaciones para el voluntariado

\begin{tabular}{lll}
\hline & Valores de Reciprocidad & \\
\cline { 2 - 3 } Expresión de valores & Valores de interés por la Comunidad & $\begin{array}{l}\text { Valores de Ayuda a un } \\
\text { Territorio Específico }\end{array}$ \\
\hline Conocimiento & $\begin{array}{l}\text { Valores de Ayuda a un } \\
\text { Colectivo Específico }\end{array}$ \\
\hline Desarrollo Profesional & \\
\hline
\end{tabular}




\begin{tabular}{ll}
\hline \multirow{2}{*}{ Realización Personal } & Estima \\
\cline { 2 - 2 } & Crecimiento Personal \\
\cline { 2 - 2 } & Realciones Sociales \\
\hline Compromiso & Compromiso con el Grupo \\
\hline
\end{tabular}

Interés en la Actividad

Fuente: Elaboración de la autora

1.2. Valores de Interés por la Comunidad. Se define como el interés comunitario por ayudar y se subdivide en dos: (A) Valores de Ayuda a un Territorio Específico, que alude al interés por brindar ayuda o apoyo a una zona geográfica específica. Al respecto, Osvaldo, estudiante universitario que pertenece a la Comunidad Wichi de La Puntana y participa del Proyecto Salud Sexual Integral-UNSa desde sus orígenes (año 2012), expresa que "uno se siente involucrado en cada situación que pasa en la comunidad porque la idea es ayudar a la gente. (...) Y si puedo ayudar de alguna manera, lo voy a hacer mediante este tipo de proyectos. Participé en un montón de proyectos (...) todos vinculados a comunidades originarias (Osvaldo-SSI). (B) Valores de Ayuda a un Colectivo Específico, donde el interés se centra en un segmento de la sociedad o grupo concreto. El relato de los estudiantes ejemplifica esta categoría: "[me motiva] brindar atención primaria a gente que, por una u otra razón, no puede llegar al hospital o el mismo sistema de salud los tiene desamparados. Ese es el principal motor" (Stella Maris-CSA); "escuchar las necesidades de las mamás, interesarse por ellas y darles respuesta son actitudes que ponen de manifiesto las más altas motivaciones humanas en nosotros" (María-NSE).

\section{Conocimiento}

Esta categoría refleja el interés por conocer, aprender y comprender el mundo, implica el desarrollo de nuevas perspectivas e interpretaciones, así como el aprendizaje y potenciación de habilidades y conocimientos. 
Involucra la oportunidad de vivenciar nuevas experiencias de aprendizaje que representan la posibilidad de ejercer los conocimientos, destrezas y habilidades que no son utilizados ni explorados en otros contextos. Expresiones tales como "(...) adquirir destrezas en el contacto con nuevas personas" (Sergio-NSE); "(...) aprender más acerca de la lactancia y transmitir la importancia de la misma a las madres, adolescentes, y familias, conocer a través de la experiencia de las madres" (Florencia-ALM), dieron lugar a esta segunda categoría general.

\section{Desarrollo Profesional}

Desarrollo Profesional concierne a los beneficios que pueden obtenerse de la participación en actividades voluntarias, en términos de mejora del currículum, experiencia pre-profesional y establecimiento de contactos profesionales que podrían actuar como puente para una futura inserción laboral. Así lo expresan los voluntarios: "(...) sumar una actividad más a mi Currículo" (Melina-NSE); "(...) poner en práctica el diálogo con las mamás para transmitirles información es algo que nos hace crecer como futuros profesionales" (Marianela-ALM); "es nuestra primera oportunidad para promover la salud, que será uno de los deberes fundamentales en nuestra futura carrera profesional" (Juan-ALM).

\section{Realización Personal}

Esta categoría motivacional -"estima" en el VFI- refiere a diversos significados y, de acuerdo con Chacón et al. (2010), incluye aspectos muy distintos relacionados con la mejora del estado de ánimo, con la autoestima o con la necesidad de mantener relaciones sociales que contribuyen al bienestar de las persons. Ello llevó a diferenciar cuatro subcategorías: una que recoge los aspectos relacionados con la estima, otra referida a la necesidad de crecer a nivel personal, una tercera relacionada con la necesidad de vincularse con otras personas y una cuarta centrada en el disfrute. Con la finalidad de abarcar estas opciones, se decidió mantener esta categoría bajo la designación de "Realización Personal" dado que 
enfatiza las posibilidades que el voluntariado ofrece para el crecimiento personal y el desarrollo psicológico que se genera mediante la participación en los proyectos estudiados.

4.1. Estima. Incluye aspectos relacionados con la valía personal que directamente buscan el aumento de la autoestima. Algunas respuestas vinculadas a esta subcategoría son: "(...) me dejó satisfecho y feliz observar cómo aquellas adolescentes apreciaban lo que hacíamos por ellas" (SergioNSE); "resulta gratificante saber que, a partir de acciones simples, es posible promover cambios" (Eliana-NSE).

4.2. Crecimiento Personal. Alude a la participación del estudiante en prácticas de voluntariado y extensión como una forma de evolucionar de manera positiva, de desarrollar todo su potencial: "(...) es muy enriquecedor, a nivel personal y a nivel comunidad (...) el aprendizaje es mutuo, yo aprendo de mis compañeros y mis compañeros aprenden de nosotros, quienes somos de pueblos originarios (Osvaldo-SSI).

4.3. Relaciones Sociales. Refleja una necesidad de establecer nuevos vínculos sociales. Al respecto, los voluntarios expresan que "la posibilidad de participar en el voluntariado sirve para (...) conocer gente nueva de distintas Facultades y ámbitos" (Ignacio-NSE); "adquirir destrezas en el contacto con nuevas personas, (...) y trabajar en grupo con otros voluntarios de otras carreras" (Sergio-NSE).

4.4. Disfrute. Incluye respuestas que aluden directamente al placer y disfrute consecuentes de la participación: "(...) disfruto mucho realizar todas las actividades porque me gusta saber que con el conocimiento que adquirimos en el módulo de lactancia se puede informar y ayudar a muchas personas" (Rocío-ALM).

\section{Compromiso Organizacional}

Esta categoría general refiere a una vinculación de tipo emocional con una organización particular. En estos casos los estudiantes participan en los proyectos de voluntariado por un compromiso con la organización y/o con el grupo. Los resultados de esta investigación no arrojan evidencia de 
motivos por compromiso institucional (una de las categorías del estudio de Chacón et al., 2010) aunque sí aparecen respuestas vinculadas al compromiso con el grupo, generalmente con las cátedras o los equipos de voluntariado y extensión.

5.1. Compromiso con el Grupo. El voluntario se siente comprometido con las personas que integran el grupo y no tanto con la organización: "(...) es un buen ámbito de trabajo, entonces así se puede trabajar bien" (NoeliaCSA); "me tocó un grupo de compañeros excelente, muy buenas personas, las reuniones son agradables, nos juntamos a planear cada taller, a coordinar los mismos sabiendo que contás con el apoyo de cada uno de ellos y sentir que todos estamos ahí para lo mismo" (Julieta-NSE).

\section{Interés en la actividad}

Finalmente, esta última categoría emergente indica que el interés está puesto en la actividad específica que se desarrolla, no en el proyecto de voluntariado en sí, ni en los fines de la organización: "(...) lo que más me motivó fue, no sólo el objetivo del voluntariado sino también el procedimiento: la realización de los cuestionarios consistiría en estar en contacto con la realidad de embarazadas y madres, enfrentarse día a día con la dura realidad de ellas" (Verónica-NSE).

Una aproximación cuantitativa de los datos indica un resultado emparentado respecto del motivo citado con mayor frecuencia. En este sentido, del análisis de 86 motivos explícitos, el 20\% refiere a Expresión de Valores; por otro lado, Conocimiento, Desarrollo Profesional y Realización Personal obtuvieron un 19\% cada uno; el 14\% se inclinó por Compromiso Organizacional, mientras que el motivo Interés por la Actividad contó con el $8 \%$ de las respuestas totales.

Otro dato importante para destacar es que del total de los estudiantes, un $40 \%$ ofreció un único motivo, frente al $60 \%$ restante que aportó dos motivos di'ferentes, e incluso más, con un valor medio de dos motivaciones por voluntario. 
El análisis de los datos permite observar que Expresión de Valores junto con Conocimiento, Desarrollo Profesional y Realización Personal son las motivaciones citadas con mayor frecuencia por los voluntarios y parecen coexistir con otras motivaciones, consideradas menos relevantes, pero muy diversas. En este sentido, los resultados de la categorización han revelado que las motivaciones expresadas por los voluntarios pueden agruparse en seis categorías y nueve subcategorías diferentes (15 en total), diez menos de las teorizadas por Chacón et al. (2010), ya que en el análisis de los datos no han surgido motivaciones por valores religiosos, de transformación social, para el ajuste social y defensa del yo, compromiso institucional e interés en la actividad con personas, como tampoco se han identificado otras categorías construidas por los autores. Respecto de la propuesta de Clary et al. (1998), las únicas motivaciones que no emergieron en el análisis fueron ajuste social y protección o defensa del yo.

\section{DISCUSIÓN Y CONSIDERACIONES}

Los estudiantes de los proyectos estudiados manifiestan tener más de un motivo para participar de las actividades, lo cual coincide con lo postulado por Allison et al. (2002) y por Chacón et al. (2010), aunque estos investigadores subrayan que los voluntarios, comúnmente, no suelen mencionar más de dos motivos.

En el estudio de Allison et al. (2002) se presenta a los voluntarios una pregunta abierta con un único espacio para la respuesta, induciendo a expresar un motivo. Contrariamente, el estudio de Chacón et al. (2010) también presenta una pregunta abierta pero sugiere a los sujetos la posibilidad de indicar múltiples motivos. En ambos casos, los datos confirmaron que, en general, los voluntarios no suelen mencionar más de dos motivaciones para el voluntariado.

Si bien algunos estudiantes de esta investigación han informado, incluso, hasta siete motivos diferentes, la media se reduce a dos, por lo que es posible confirmar los resultados encontrados por los autores referidos, y pensar que detrás de la acción voluntaria pueden coexistir hasta dos motivos 
distintos que llevan al estudiante a implicarse y permanecer en proyectos de extensión y voluntariado por largos períodos de tiempo.

Es preciso realizar una diferenciación importante respecto de los estudios mencionados. En primer lugar, tanto Allison et al. (2002) como Chacón et al. (2010) han estudiado las motivaciones para el voluntariado mediante una pregunta abierta en una muestra numerosa de sujetos voluntarios, de edades variadas, pertenecientes a diversas organizaciones. En este caso particular se han analizado las motivaciones para el voluntariado en una muestra pequeña de estudiantes universitarios que participan de proyectos de voluntariado y extensión de la Universidad en la cual están cursando sus estudios superiores. Además, los datos fueron recogidos, no mediante la realización de una pregunta abierta y directa, sino que la información fue relevada a partir de un análisis documental de los relatos de experiencia y de algunas entrevistas en profundidad, lo cual no dio lugar a una respuesta acabada respecto de los motivos por los cuales están implicados; tales motivos, por el contrario, fueron inferidos por la investigadora a partir del análisis de las narraciones de sus experiencias subjetivas.

No obstante esta diferenciación a nivel muestra e instrumentos, los resultados encontrados parecen coincidir, en parte, con los de los autores referidos, especialmente en relación con la manifestación de, al menos, dos motivos para ser voluntarios. Desde la perspectiva funcionalista (CLARY et al., 1998), diferentes funciones psicológicas pueden ser satisfechas a través de la implicación del individuo en la práctica del voluntariado, lo que permite entender que, si bien muchas personas participan en una misma actividad, los motivos por los cuales se implican pueden ser sustancialmente diferentes. Respecto de las motivaciones de los alumnos, los resultados muestran que la categoría Expresión de Valores fue la más citada, junto con las funciones del voluntariado como fuente de Conocimiento, Desarrollo Profesional y Realización Personal.

En términos motivacionales, algunas funciones para el voluntariado obtienen una similar importancia en diversos grupos etarios. Expresión de 
Valores, por ejemplo, recibe generalmente altas puntuaciones tanto en individuos jóvenes (18 a 24 años) como en sujetos mayores de 65 años de edad (CLARY et al., 1998). Sin embargo, algunas motivaciones varían: las necesidades que giran en torno al desarrollo profesional y a la adquisición de nuevas habilidades, competencias y conocimientos parecieran ser más importantes en los voluntarios más jóvenes que en las personas mayores (CLARY et al., 1996; DÁVILA, DÍAZ-MORALES, 2009; OKUN, SCHULTZ, 2003).

En relación con Desarrollo Profesional, los resultados son coincidentes con los encontrados por otros autores (CLARY et al., 1996; DÁVILA, DÍAZMORALES, 2009; GIDRON, 1978; HOLDSWORTH, 2010; OKUN et al., 1998; OKUN, SCHULTZ, 2003) y refuerzan la idea de que los sujetos jóvenes tienden a inviertir mayor tiempo y energías en la adquisición de nuevos aprendizajes o experiencias relacionadas con la carrera. Ello podría deberse al hecho de que, cuando adultos, las personas adquieren nuevas responsabilidades laborales y familiares $y$, con ello, alcanzan un cierto nivel de estabilidad y experiencia de vida. Los individuos más jóvenes tienden a valorar metas y objetivos orientados a optimizar su futuro profesional. De acuerdo con Erikson (1980) en los inicios de la edad adulta, los jóvenes se enfrentan con ciertos conflictos normativos, de los cuales se puede mencionar la necesidad de consolidar la propia identidad, a partir de la resolución de tareas tales como el desarrollo de un plan de su futuro, consolidar su autoestima, desenvolver nuevas competencias y asumir nuevas responsabilidades. Atendiendo a ello, se puede pensar que el hecho de que los estudiantes hayan destacado la función de Desarrollo Profesional, Conocimiento y Realización Personal como otros motivos para el voluntariado, además de Expresión de Valores, podría responder a esta necesidad psicológica del individuo de vislumbrar, en la práctica del voluntariado, una posibilidad de aprender, de insertarse en el mercado de trabajo y de realizar contactos que puedan contribuir al desarrollo de su carrera profesional, posibilitándole proyectar su futuro y ganar, de esa forma, una mayor competitividad en el terreno laboral.

De acuerdo con los postulados del Enfoque Funcionalista (CLARY et al., 1998) se puede afirmar que la participación en acciones de voluntariado 
está sujeta al grado de coincidencia entre las inquietudes motivacionales de los jóvenes universitarios con situaciones que pueden satisfacerlas. Es decir, los estudiantes encuentran oportunidades de respuesta a sus necesidades motivacionales en las actividades que ofrecen los proyectos de extensión y voluntariado en los que están insertos. En este sentido, una variable a considerar está relacionada con la elección de la carrera y con el modo en cómo determinadas carreras o áreas del conocimiento podrían configurar la participación y valoración de los estudiantes en diferentes actividades que se ajusten a sus necesidades. Las diferentes facultades pueden asumir posturas diversificadas frente al compromiso y a la responsabilidad con la sociedad, y propiciar -o inhibir- nuevas formas de participación e implicación social, así como también promover en los individuos actitudes prosociales o antisociales.

En el contexto de las universidades observadas, tanto la UNSA como la UNT poseen una fuerte tradición extensionista y, del mismo modo, los proyectos analizados vienen desarrollándose -con un mínimo de cuatro años- como prácticas exitosas de vinculación social universitaria, con evaluaciones positivas respecto de su gestión. Se puede pensar, por lo tanto, que los espacios disponibilizados desde las diverentes cátedras de las Facultades de Salud y Medicina están brindando oportunidades de respuesta a las inquietudes motivacionales de los estudiantes que participan de tales espacios.

Cuando se indaga directamente a los voluntarios sobre las razones por las cuales son voluntarios, generalmente suelen mencionar motivos de tipo altruista: ayudar al que más necesita. La preocupación por los otros es, a menudo, una característica sobresaliente de los voluntarios que los distingue de los no voluntarios, y puede predecir si los sujetos sustentarán su actividad durante períodos más prolongados o si desistirán antes de concluir el primer año de participación (CLARY et al., 1998). Incluso los resultados de esta investigación apuntan en esa dirección. Sin embargo, se ha observado que los sujetos pueden perseguir, también, metas personales o egoístas (CHACÓN et al., 2010; CLARY et al., 1998; ORTIZ, 2013) en el sentido de 
buscar, intencionalmente, algún rédito a cambio. Se puede pensar que ambas razones -altruistas y egoístas- coexisten en una misma conducta voluntaria (HASKI-LEVENTHAL, 2009), lo cual conduce a adoptar una perspectiva donde el comportamiento voluntario forma parte de un proceso cognitivo y social que implica la percepción de los costos asociados (en términos del tiempo y la energía de las cuales dispensará el voluntario para desarrollar una determinada actividad) $y$, fundamentalmente, la construcción de beneficios personales que pueden obtenerse de la acción voluntaria. Desde esta perspectiva, la conducta voluntaria no refiere únicamente a un simple acto de generosidad en respuesta a una necesidad social, sino que también puede entenderse como una competencia sociocognitiva de la persona voluntaria que, consciente y deliberadamente, busca construir beneficios en un tipo de respuesta donde aparentemente sólo se visualizan costos implicados (ORTIZ, 2013).

En coherencia con esta premisa, los estudiantes de la investigación han mencionado motivaciones heterocentradas (expresión de valores) y también autocentradas (conociminento, desarrollo profesional y realización personal). El voluntariado, por lo tanto, es visto como un contexto donde el individuo puede manifestar libremente sus valores personales relacionados con el altruismo y el humanitarismo, pero también representa una oportunidad para explorar nuevas experiencias de aprendizaje y aplicar los conocimientos, destrezas y habilidades que en otros espacios universitarios no podría poner en práctica. En este sentido, se destaca un número creciente de estudiantes universitarios, de carreras del área de la salud, que se ofrecen como voluntarios en los diferentes proyectos, puesto que es allí donde pueden ejercer y "entrenar" los conocimientos y competencias adquiridos, aprender más sobre la propia práctica y ganar una mayor experiencia para un futuro profesional.

Desarrollar esta perspectiva del voluntariado puede tener efectos positivos sobre la implicación voluntaria de los estudiantes puesto que los sujetos pocas veces son conscientes de los conocimientos, habilidades y valores que se adquieren a través de esa páctica (BURNS et al., 2006, MATO, 
2013). Y, en este sentido, "Ios contextos académicos pueden propiciar espacios y experiencias para adquirir conciencia de las consecuencias positivas de la acción voluntaria para la vida del otro, de la comunidad, también y fundamentalmente, para la vida del propio sujeto" (ORTIZ, 2013, p. 72).

El voluntariado es una forma organizada de participación de ciudadanos que asumen libremente un triple compromiso: cooperar en la identificación y denuncia de situaciones injustas, buscar soluciones a los problemas que afectan a la sociedad en su conjunto y colaborar activamente en la mejora de actividades culturales y sociales (MARTínEZ, 2008). Las prácticas de voluntariado y extensión, además de ser un aporte significativo para la sociedad, se transforman en espacios de aprendizaje, donde lo teórico y lo empírico dialogan y los contenidos académicos pueden aprenderse en interacción con contenidos derivados de una realidad social próxima que, muchas veces, los propios alumnos desconocen.

Resulta de interés, por lo tanto, pensar en todas estas cuestiones de modo a promover una mayor participación del estudiante en proyectos de voluntariado o extensión universitaria que puedan atender a los intereses, necesidades e inquietudes motivacionales de cada persona en particular $y$, que a su vez, puedan propiciar espacios de prácticas donde los individuos sean capaces de recrear y fortalecer sus conocimientos, poniéndolos al servicio de una causa social.

Conocer, hacia adentro, qué es lo que motiva a los estudiantes a participar en acciones de voluntariado se torna un recurso crucial para los equipos docentes e investigadores que promueven el desarrollo de experiencias de vinculación social universitaria desde diferentes espacios de formación. Ello disponibilizaría información relevante que permita: (a) repensar la oferta y las prácticas de extensión que actualmente se desarrollan en las universidades; (b) adecuar estas prácticas, no sólo a la necesidades del medio social, sino también a las necesidades e inquietudes 
motivacionales de los estudiantes; (c) lograr una mayor participación, compromiso social y permanencia en los diferentes proyectos.

\section{REFERENCIAS}

ALLISON, L.; OKUN, M.; DUTRIDGE, K. Assessing Volunteer Motives: A Comparison of an Open-ended Probe and Likert Rating Scales. Journal of Community \& Applied Social Psychology, v. 12, n. 4, p. 243-255, 2002. http://dx.doi.org/10.1002/casp.677

ARIAS, G.; TARZIBACHI, M. Repensando la pertinencia social de la Universidad: voluntariado, organizaciones sociales y comunidad. In: ARIAS, G. (Org.). Participación e Innovación en Educación Superior. Para que el conocimiento nos sirva a todos. Buenos Aires: Ministerio de Educación, Ciencia y Tecnología de la Nación, 2007, p. 43-55.

BRUYERE, B.; RAPPE, S. Identifying the motivations of environmental volunteers. Journal of Environmental Planning and Management, v. 50, n. 4, p. 503-516, 2007. https://doi.org/10.1080/09640560701402034

BURNS, D.; REID, J.; TONCAR, M.; FAWCETT, J.; ANDERSON, C. Motivations to Volunteer: the Role of Altruism. International Review on Public and Non Profit Marketing, v. 3, n. 2, p. 79-91, 2006. https://doi.org/10.1007/BF02893621

CHACÓN, F.; GUTIÉRREZ, G.; SAUTO, V.; VECINA, M. L.; PÉREZ, A. Volunteer Functions Inventory: A systematic review. Psicothema, v. 29, n. 3, p. 306-316, 2017. https://doi.org/10.7334/psicothema2016.371

CHACÓN, F.; PÉREZ, T.; FLORES, J.; VECINA, M. L. Motivos del Voluntariado: Categorización de las Motivaciones de los Voluntarios Mediante Pregunta Abierta. Intervención Psicosocial, v. 19, n. 3, p. 213-222, 2010. http://scielo.isciii.es/scielo.php?script=sci_arttext\&pid=S1 132$05592010000300002 \&$ lng $=$ es\&nrm=iso

CLARY, E. G.; SNYDER, M. A functional analysis of altruism and prosocial behavior: The case of volunteerism. In Margaret, S. (Ed.), Prosocial behavior. Review of personality and social psychology, 1991, p. 119-148. Thousand Oaks, CA: Sage Publications, Inc.

CLARY, E., SNYDER, M., \& STUKAS, A. Volunteers' motivations: Findings from a national survey. Nonprofit and Voluntary Sector Quarterly, v. 25, n. 14, p. 485505, 1996. https://doi.org/10.1177/0899764096254006

CLARY, G.; SNYDER, M. The Motivations to Volunteer: Theoretical and Practical Considerations. Current Directions in Psychological Science, v. 8, n. 5, p. 156159, 1999. https://doi.org/10.1111/1467-8721.00037 
CLARY, G.; SNYDER, M.; RIDGE, R.; COPELAND, J.; STUKAS, A.; HAUGEN, J.; MIENE, P. Understanding and assessing the motivations of volunteers: A functional approach. Journal of Personality and Social Psychology, v. 74, n. 6, p. 1516-1530, 1998. http://dx.doi.org/10.1037/0022-3514.74.6.1516

CNAAN, R.; GOLDBERG-GLEN, R. Measuring motivations to volunteer in human services. Journal of Applied Behavioral Science, v. 27, n. 3, p. 269-284, 1991. https://doi.org/10.1177/0021886391273003

DÁVILA, M. C.; DÍAZ-MORALES, J. Age and motives for volunteering: Further evidence. Europe's Journal of Psychology, v. 5, n. 2, p. 82-95, 2009. https://doi.org/10.5964/ejop.v5i2.268

ERIKSON, E. Identity and the Life Cycle. New York: W. W. Norton \& Company, 1980.

FIGUEIRA, C. Bem-estar nos estudantes do ensino superior: papel das exigências e dos recursos percebidos no contexto académico e das atividades de voluntariado. 2013. 232f. Tese (Doutorado em Psicología) Universidade de Lisboa, Lisboa, 2013.

FINKELSTIEN, M. Intrinsic vs. extrinsic motivational orientations and the volunteer process. Personality and Individual Differences, v. 46, n. 5-6, p. 653658, 2009. https://doi.org/10.1016/j.paid.2009.01.010

GIDRON, B. Volunteer Work and its Rewards. Volunteer Administration, v. 11, n. 3, p. 18-31, 1978.

https://www.researchgate.net/profile/Benjamin_Gidron/publication/12989435 _Volunteer_Work_and_Its_Rewards/links/0912f50872a72a413a000000.pdf

HASKI-LEVENTHAL, D. Altruism and Volunteerism: The perceptions of altruism in four disciplines and their impact on the study of volunteerism. Journal for the Theory of Social Behaviour, v. 39, n. 3, p. 271-299, 2009.

http://dx.doi.org/10.1111/j.1468-5914.2009.00405.x

HOLDSWORTH, C. Why Volunteer? Understanding motivations for student volunteering. British Journal of Educational Studies, v. 58, n. 4, P. 421-437, 2010. https://doi.org/10.1080/00071005.2010.527666

HOULE, B., SAGARIN, B., \& KAPLAN, M. A Functional Approach to Volunteerism: Do Volunteer Motives Predict Task Preference? Basic and Applied Social Psychology, v. 27, n. 4, p. 337-344, 2005.

MARTíNEZ, M. "Aprendizaje servicio y construcción de ciudadanía activa en la universidad: la dimensión social y cívica de los aprendizajes académicos", In: Martínez, M., Aprendizaje servicio y responsabilidad social de las universidades, España, Limpergarf, S.L, pp. 7-11, 2008.

MATO, D. Contribución de experiencias de vinculación social de las universidades al mejoramiento de la calidad académica y factores que 
limitan su desarrollo y valoración institucional. Avaliação, Campinas, v. 18, n, 1, p. 151-180, 2013. http://www.redalyc.org/html/2191/219125744009/

NICHOLLS, J.; SCHIMMEL, K. Match/Mismatch of the College Business Student Service-Learning Experiences: Drivers of Perceived Attitude Change, Satisfaction, and Future Volunteering Intentions. Journal of Higher Education Theory and Practice, v. 12, n. 6, p. 91-99, 2012.

OKUN, M.; SCHULTZ, A. Age and motives for volunteering: Testing hypotheses derived from socioemotional selectivity theory. Psychology and Aging, v. 18, n. 2, p. 231-239, 2003. http://dx.doi.org/10.1037/0882-7974.18.2.231

OKUN, M.; BARR, A.; HERZOG, A. Motivation to volunteer by older adults: A test of competing measurement models. Psychology and Aging, v. 13, n. 4, p.

608-621, 1998. http://pages.stern.nyu.edu/ kbrabazo/Evalrepository/Repository-Articles/motivation\%20to\%20voluneer.pdf

ORTIZ, A. Creencias Motivacionales y Actitudes frente al Voluntariado: Un estudio con jóvenes universitarios en Portugal. 2013. 425f. Tese (Doutorado em Educação) - Universidade de Lisboa, Lisboa, 2013. http://hdl.handle.net/10451/9805

TAPIA, N. Aprendizaje y servicio solidario en la misión de la Educación Superior. In: GONZÁLEZ, A.; MONTES, R. El Aprendizaje-Servicio en la Educación Superior: Una mirada analítica desde los protagonistas. Buenos Aires: EUDEBA, 2008, pp. 5-11.

WU, J.; WING LO, T.; LIU, E. S. Psychometric properties of the volunteer functions inventory with Chinese students. Journal of Community Psychology, v. 37, n. 6, p. 769-780, 2009. http://dx.doi.org/10.1002/jcop.20330

Recebido em: 02 de abril de 2018 Aprovado em: 08 de outubro de 2018 